\title{
The balance between protective and pathogenic immune responses in the TB-infected lung
}

\author{
Ian M Orme ${ }^{1}$, Richard T Robinson ${ }^{2} \&$ Andrea M Cooper ${ }^{3}$
}

\begin{abstract}
Tuberculosis is a disease of the lung, and efficient transmission is dependent on the generation of a lesion in the lung, which results in a bacterium-laden cough. Mycobacterium tuberculosis (Mtb) is able to manipulate both the innate and acquired immune response of the host. This manipulation results in an effective CD4+ T cell response that limits disease throughout the body but can also promote the development of progressively destructive lesions in the lung. In this way Mtb infection can result in an ambulatory individual who has a lesion in the lung capable of transmitting Mtb. The inflammatory environment within the lung lesion is manipulated by Mtb throughout infection and can limit the expression of acquired immunity by a variety of pathways.
\end{abstract}

Tuberculosis (TB) has been both instructing and confounding immunologists and vaccinologists for many years. Bacteria can be detected within the lung tubercles using a stain developed more than 100 years ago, but it is not yet possible to define the mechanisms that generate the tubercle or, indeed, the specific pathways that mediate bacterial killing within this tubercle.

The currently held paradigm of how the body controls infection with M. tuberculosis (Mtb) is that macrophages recognize and take up the bacterium, whereupon it proliferates within this phagocyte until either the cell dies or is instructed by an antigen-specific T cell to kill or at least limit the growth of the bacterium. The current widely used vaccine, being a live attenuated strain of the related Mycobacterium bovis (BCG), mimics this lifestyle by infecting host phagocytes and driving antigen-specific $\mathrm{T}$ cell responses. This vaccine is highly effective at limiting disseminated disease in children ${ }^{1}$ but works erratically to protect against pulmonary disease $\mathrm{e}^{2}$. Although it is clear that antigen-specific $\mathrm{CD} 4^{+} \mathrm{T}$ cells and macrophage-activating cytokines are required for the control of $\mathrm{TB}^{3}$, it is not clear that simply inducing more of these T cells or cytokines will result in improved protection ${ }^{4}$. Our current failure to improve on BCG lies in the fact that we do not yet understand what constitutes a protective immune response to TB in the lung, nor do we know the extent to which the phenotype of antigen-specific $\mathrm{CD} 4^{+} \mathrm{T}$ cells contributes to this protective response. Another potential confounder is that Mtb seems to be adept at manipulating the human immune response.

\footnotetext{
${ }^{1}$ Mycobacteria Research Laboratories, Department of Microbiology, Immunology and Pathology, Colorado State University, Fort Collins, Colorado, USA. ${ }^{2}$ Department of Microbiology and Molecular Genetics, Medical College of Wisconsin, Milwaukee, Wisconsin, USA. ${ }^{3}$ Trudeau Institute, Inc., Saranac Lake, New York, USA. Correspondence should be addressed to A.M.C. (acooper@trudeauinstitute.org).
}

Received 24 September; accepted 5 November; published online 18 December 2014; doi:10.1038/ni.3048
Here we describe, using the mouse model as a tool, the factors contributing to the early interaction between host and pathogen. We will also discuss the phenotype of antigen-specific $\mathrm{T}$ cells and how this is important to the ability of the $\mathrm{T}$ cell to function within the Mtb-defined inflammatory lesion within the lung.

\section{The unique interaction between Mtb and humans}

Mtb and humans seem to have co-evolved during the period before the migration of humans out of Africa ${ }^{5}$, and this has prompted the speculation that Mtb has had considerable opportunity to develop tools with which to manipulate the human immune response to its own advantage. Indeed, the rate of Mtb mutation is the same regardless of generation time in the host ${ }^{6}$, which suggests that although there is significant genomic stability within Mtb, mutation occurs throughout the interaction between host and pathogen. Importantly, a substantial portion of the Mtb genome is devoted to secretion systems, which contain the majority of immunodominant antigens ${ }^{7}$. Immunodominant epitopes have been suggested to be highly conserved ${ }^{8}$, but there is evidence of diversity in these epitopes ${ }^{9}$. Regardless of the diversity of the epitopes, the genetic investment of the bacterium in human $\mathrm{T}$ cell epitopes associated with secretion systems suggests that activated, antigen-specific $\mathrm{T}$ cells are required for essential elements of the Mtb life cycle. Mtb also invests strongly in the generation of a variety of molecules on its cell surface, and these are capable of influencing the innate immune response. An example of a highly studied manipulator of host inflammation is trehalose dimycolate (TDM, also known as cord factor), which binds members of the Clec 4 family of C-type lectins (MCL and Mincle) on host myeloid cells to drive granuloma formation in mice ${ }^{10-13}$, and within which alterations in structure result in substantial changes in inflammatory capacity ${ }^{14-16}$.

One could hypothesize that the need to be transmitted between humans (the only environmental niche for $\mathrm{Mtb}$ ) requires that the Mtb-infected individual develop a lung lesion capable of mediating 
Figure 1 Events occurring early in infection influence infection outcome. Mtb bacteria enter the lung and establish infection in the alveoli. High numbers of neutrophils in the blood at the time of exposure are associated with lower likelihood of infection. If infection is established, migratory cells take bacteria to the draining lymph node in a CCR2dependent manner. In the draining lymph node, T cells become activated as a result of $\mathrm{MHC}$ class II expression on dendritic cells. During the time taken for the migration of the bacteria to the draining lymph node (7-9 d), permissive macrophages are recruited to the lung, and bacteria proliferate within these cells. Bacteria also disseminate to other organs, but this is delayed relative to the arrival of bacteria in the lung. Owing to this delay in seeding of other organs by the disseminating bacteria, the circulating activated T cells generated in the lung-draining lymph node will be ready to populate these newly infected organs. Owing to the concurrent arrival of disseminating bacteria and activated T cells in these other organs, there is reduced time available for the accumulation of noninfected macrophages, and the T cells will be better able to interact with and activate the infected macrophages. In this way, the impact of the acquired response on control of bacterial growth in the lung is compromised relative to other infected organs within the infected individual.

transmission but remain ambulatory enough to come in contact with many other individuals in order to successfully transmit infection. This requirement could explain the focus of Mtb on manipulating both the innate and acquired immune response. Within this framework, a feasible working model is that Mtb initiates recruitment of permissive phagocytes into the lung while limiting the early induction and recruitment of $\mathrm{T}$ cells capable of redirecting the permissive phagocytes to a bactericidal state. This would then result in a permissive environment wherein the bacterium can grow without constraint. However, if this type of response were to occur throughout the body, the host would rapidly become sick and die, thereby failing to transmit infection to further potential hosts. Thus, expression of strong T cell immunity early in Mtb infection would compromise establishment of a permissive lesion in the lung, but this same $\mathrm{T}$ cell immunity is crucial in limiting bacterial growth in the non-lung organs to maintain the ambulatory state of the person infected (Fig. 1). In support of the concept that the T cell response is required for a 'successful' disease profile-for Mtb rather than the host-it is clear that in the absence of a strong $\mathrm{T}$ cell response, such as in $\mathrm{T}$ cell depletion during HIV infection, humans develop a disseminated and rapidly fatal disease ${ }^{17}$. The potential for an overwhelming inflammatory response to compromise effective bacterial control has also been suggested by histological studies of human lungs in the pre-drug era. These studies showed that in the same patients, larger lesions progressed and smaller ones resolved, and the lesions arising from disseminating bacteria were more circumscribed and contained fewer bacteria ${ }^{18}$. This working model provides a rationale for why the majority of Mtb disease occurs within the lung (Fig. 1). It also suggests that events that occur early in infection could affect the development of disease.

\section{Early events after infection}

It is thought that Mtb enters the lung via small aerosolized droplets exhaled by people who are infected and have high bacterial burdens and disrupted lesions that have accessed the airways. It is not clear what transmissible Mtb bacteria look like or if all of the Mtb bacteria in an infected person are equally able to transmit disease. In the absence of this information, most studies are done with cultured Mtb, and it is likely that subtleties associated with transmission have not yet been fully modeled.

Although deposition of Mtb in the alveoli is thought to be the primary route of entry, it has been shown that the bacterium can infect human lung epithelial cells and that these infected cells drive activation

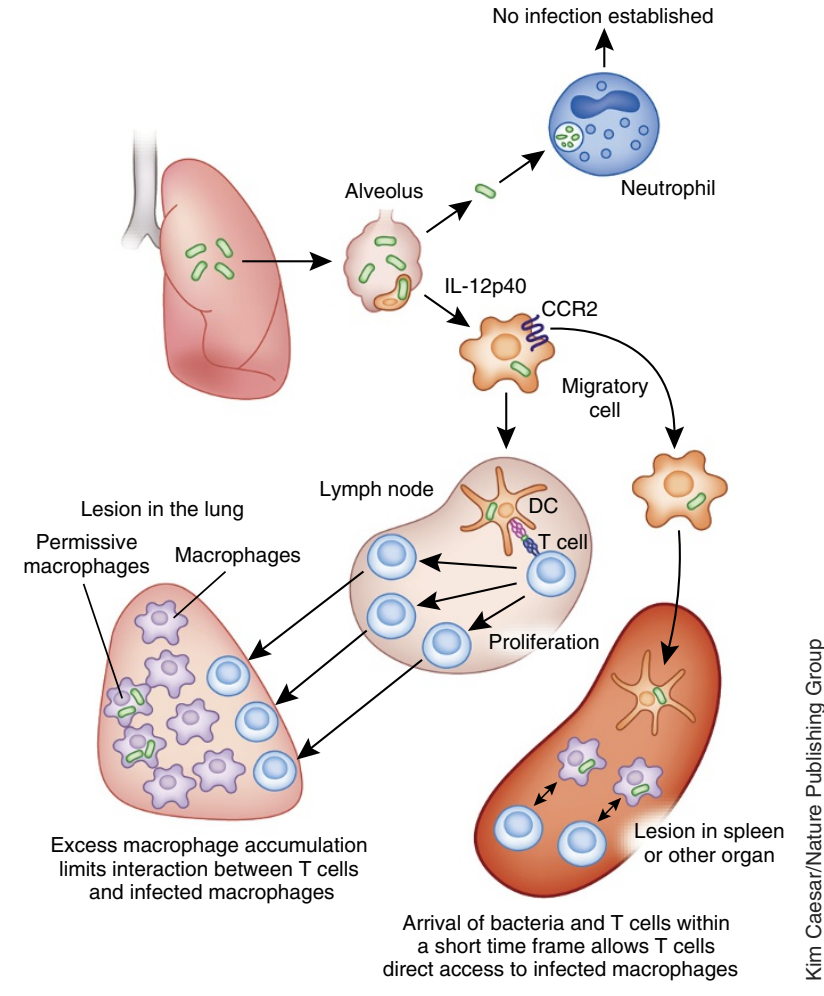

of mucosal associated invariant T (MAIT) cells, which suggests a surveillance role for these cells in recognizing primary infection ${ }^{19}$. Deposition on the epithelium and activation of MAIT cells may result in an unsuccessful infection process by limiting early bacterial growth and by alerting the acquired response to infection. Other defense mechanisms inherent to the airway are also likely to have a crucial role in limiting infection after initial exposure. Innate lymphoid cells such as invariant natural killer T cells ( $i$ NKT cells), which produce macrophage-activating GM-CSF ${ }^{20}$, or $\gamma \delta \mathrm{T}$ cells, which respond rapidly to mycobacterial antigens and express a variety of effector functions ${ }^{21}$, are both potential players in limiting early infection. Current studies of associations between genetic polymorphisms in the innate immune system and risk of TB suggest that there is a potentially wide variety of innate responses that have protective roles in early infection ${ }^{22}$. In contrast, the immune defects underlying the spectrum of conditions known as Mendelian susceptibility to mycobacterial disease in humans highlights the need for macrophage activation via interleukin 12 (IL-12)-dependent interferon- $\gamma($ IFN- $\gamma$ ) signaling in resistance to mycobacterial disease $\mathrm{e}^{23,24}$.

Although the epithelium can become infected, macrophages in the alveoli are thought to be the primary target for Mtb once it enters the lung, and the interaction between Mtb and these cells is thought to define the subsequent progression of infection. It is crucial to the understanding of the earliest events in the lung to define the impact of the environment encountered by the bacterium. Some work has identified a role in this for the lung hydrolases. These hydrolases are present in human surfactant and alter the envelope of the invading Mtb, thereby reducing the ability of the bacterium to interact with and grow within alveolar macrophages ${ }^{25}$. Despite a perceived primary role in protection against Mtb infection, depletion of macrophages before a lethal infection with Mtb results in improved survival in the mouse model ${ }^{26}$; however, specific depletion of activated macrophages is detrimental ${ }^{27}$. These data support the idea that there are different cellular niches in the early accumulating phagocytes that respond 
to Mtb infection in the lung (Fig. 1). In strong support of this concept, studies in zebrafish have demonstrated the ability of Mtb to manipulate the earliest recruitment of macrophages. This activity has been linked to the capacity of the bacterium to express lipids on its cell surface. Specifically, expression of the Mtb molecule phthiocerol dimycoceroserate (PDIM) limits innate recognition of Mtb, thereby reducing recruitment of Toll-like receptor (TLR)-activated macrophages $^{28}$. In addition to this subversion of the pathogen-recognition pathway, other lipids on the Mtb surface-the phenolic glycolipids (PGLs) - drive recruitment of permissive macrophages via a pathway that depends on the chemokine CCL2 and the chemokine receptor CCR2 (ref. 28). One other defense pathway may be neutrophil recruitment. Specifically, studies in contacts of people with pulmonary TB found that the risk of infection (not disease) was inversely associated with the number of neutrophils in the blood of the individuals tested and that the neutrophils were themselves antibacterial ${ }^{29}$ (Fig. 1). Although this study did not directly define the role of neutrophils in early control of infection, it suggests a working model for an underlying mechanism whereby some people do not show signs of infection despite heavy exposure.

The above observations support the hypothesis that the outcome of Mtb infection in the lung depends on the initial environment encountered by the bacterium. This environment depends on the cells it first encounters, and these, in turn, may depend the genetics of the host ${ }^{30}$. These early events can also be affected by the general health of the lung (such as the presence of ongoing viral infection or smokinginduced lung damage), and it is likely that the stochastic nature of these events contributes to the wide variation in outcomes-ranging from no sign of infection to the development of primary progressing $\mathrm{TB}-$ for people exposed to the same index case.

\section{Events that contribute to dissemination}

Regardless of the initial interaction between Mtb and the innate defense mechanisms of the lung, Mtb is known to migrate from the lung throughout the body; however, it is rare for signs of disease to appear outside the lung. Disseminated disease is seen in infants and in people infected with HIV and is thought to result from the absence of effective acquired immunity ${ }^{31,32}$. The initiation of the acquired response is preceded by the appearance of bacteria in the draining lymph nodes ${ }^{33,34}$ and is associated with the expression of Mtb antigen within this organ ${ }^{35}$. The acquired response is also delayed relative to the initiation of infection ${ }^{3}$.

It has been postulated that the alveolar macrophage initiates the granulomatous response to Mtb in the lung, and that the function of this response is to wall off the pathogen and limit the spread to other organs. It is likely, however, that there are waves of recruitment to the infection site, including the local accumulation of tissue macrophages, the accumulation of inflammatory macrophages and the migration into and out of the lesion by migratory cells, which carry bacteria to other organs ${ }^{36}$. Some data suggest that the inflammatory response to Mtb is a dynamic process that potentially involves the migration of cells into and out of the granuloma in a continuous fashion ${ }^{37}$. Specifically, granulomata transplanted from an infected donor mouse into a new host release cells expressing the integrin CD11c into that host ${ }^{38}$, labeled macrophages also migrate out of the granuloma in the zebrafish ${ }^{39}$ and mouse ${ }^{40}$ models, and T cells migrate readily through the granuloma ${ }^{40,41}$. The earliest movement from the lung to the draining lymph node has been ascribed to dendritic cells, as these are heavily infected in the lung and in the lymph node ${ }^{35}$. Dendritic cells infected with Mtb deposited into the lung also migrate from this organ to the draining lymph node ${ }^{42}$, and this migration is dependent on the expression of IL-12p40 (ref. 43) (Fig. 1) and the receptor IL-12R $\beta 1$ (ref. 44) in the migrating dendritic population. In addition, Mtb induces an alternative splice variant of IL-12R $\beta 1$ in the CD11c-expressing population in the lung at the same time that the migration of Mtb from the lung to the draining lymph node occurs, and this alternatively spliced receptor is required for optimal stimulation of antigen-specific $\mathrm{T}_{\text {cells }}{ }^{44}$. Studies have implicated the tumor necrosis factor (TNF) receptor TNFRp75 as a specific downregulator of the availability of bioactive TNF. In the absence of this molecule, mice infected with Mtb have more bioactive TNF and IL-12p40, more IL-12p40-dependent migration of dendritic cells to the draining lymph node, more $\mathrm{T}$ cell activation and lower bacterial burden ${ }^{45}$. This suggests that TNFRp75 acts a sink for TNF and downregulates the protective response to Mtb in the lung. Work using chimera models and depletion of specific cells during the early stages of infection has implicated inflammatory monocytes in the actual movement of the Mtb from the lung to the draining lymph node. Importantly, although inflammatory monocytes expressing CCR2 are required for the movement of bacteria to the lymph node, it is major histocompatibility complex (MHC) class II-expressing dendritic cells that are required for T cell activation ${ }^{46}$ (Fig. 1).

These findings suggest that migration of Mtb away from the primary site of infection-the lung-occurs despite the generation of granulomatous responses and that the bacterium uses host cells to mediate this migration. The problem with this migration is that it occurs only 7-9 days after initial infection. As a consequence, there is a delay of up to 18-20 days before antigen-specific $\mathrm{T}$ cells accumulate to sufficient numbers in the lung to stop bacterial growth ${ }^{33-35,47}$. Even after vaccination, when a population of circulating memory $\mathrm{T}$ cells is present, this accumulation of antigen-specific T cells is accelerated by only five days, and Mtb bacteria still have 15 days in which to define the lung lesion before $\mathrm{T}$ cells arrive ${ }^{48}$.

\section{Recruitment of $\mathrm{T}$ cells to the lung environment}

Once the acquired response has been initiated, it must migrate from the circulation into the parenchyma of the lung and then into the infected site, which is composed largely of macrophages and dendritic cells ${ }^{49,50}$ expressing a variety of effector functions ${ }^{51}$. This predominantly mononuclear environment is thought to be maintained by the expression of the protective cytokine IFN- $\gamma^{52}$. IFN- $\gamma$ limits the survival of neutrophils ${ }^{53}$ and reduces the expression of IL-17 and recruitment of neutrophils by acting on radio-resistant cells in the lung $^{54}$. Indeed, when protective immunity is ineffective, one of the most dramatic outcomes is the accumulation of neutrophils within the lung. Infected neutrophils can be seen in airway samples from patients with active disease ${ }^{55}$, and a neutrophil-driven signature is also associated with active disease in peripheral blood analysis ${ }^{56}$. Thus, although neutrophils might have a protective role immediately after exposure ${ }^{29}$, they are strongly associated with the loss of Mtb containment and the progression to active disease.

One way in which neutrophils could interfere with the protective response to $\mathrm{Mtb}$ is to limit the interaction between infected phagocytes and antigen-specific $\mathrm{T}$ cells. It has been assumed that $\mathrm{T}$ cells and infected macrophages must interact for efficient control of $\mathrm{Mtb}$, and this was shown by the use of a chimeric mouse model wherein some phagocytes expressed MHC class II and others within the same lung environment did not. In this model, if the T cell and infected macrophage could not interact via MHC class II, then the number of Mtb bacteria in the infected macrophage was higher than in those infected macrophages able to express MHC class II ${ }^{57}$ (Fig. 2). This observation ties in with other work in which the migration of lymphocytes 
Figure 2 Location of antigen-specific T cells in the lung is dependent on chemokines and chemokine receptor expression. To interact with infected macrophages to effectively control mycobacterial growth, T cells must leave the vasculature and migrate toward the macrophage-dominated lesion. Expression of CXCR3 is associated with the ability to enter the parenchyma, and CXCR5 is required for the T cells to migrate toward the infected site within the parenchyma. Focused expression of CXCL13 in the lung, which is associated with the generation of $\mathrm{B}$ cell follicles close to the inflamed site, is required for optimal focusing of $T$ cells away from the perivascular region and toward the macrophage-dominated lesion. The combined expression of chemokines by the inflamed tissue and of chemokine receptors by effector T cells is crucial for the optimal control of infection in the lung.

in the granuloma is compromised in the absence of the homeostatic chemokines CXCL13, CC19 and CCL21 (ref. 58). In this model, the $\mathrm{T}$ cells were not able to enter the macrophage areas in the infected lung effectively-even though they were in the parenchyma and able to produce cytokines-and this was temporally associated with reduced control of bacterial growth ${ }^{58}$ (Fig. 2). Expression of homeostatic chemokines and the associated development of lymphoid follicles have been shown in the Mtb-infected lung in mouse and nonhuman primate (NHP) models and in humans, and they are associated with a positive outcome $e^{58-61}$. The function of these follicles is not yet fully defined, but in their absence there is a reduction in focused CXCL13 expression in the follicles and an increase in lymphocyte perivascular accumulation ${ }^{62}$, as well as diminished Mtb containment in the absence of CXCL13 and its receptor CXCR5 on T cells ${ }^{58,61,62}$ (Fig. 2). It seems, therefore, that CXCL13 and CXCR5 can act to recruit $\mathrm{T}$ cells to the infected macrophage areas, either by generation of a gradient associated with the location of induced lymphoid follicles or as a signal that simply releases the effector $\mathrm{T}$ cells from the perivascular region. These data support the hypothesis that the granuloma is a dynamic lesion that has the capacity to influence $\mathrm{T}$ cell migration both locally and distally.

Although migration of $\mathrm{T}$ cells in the parenchyma is crucial for optimal interaction with infected macrophages and the control of bacterial growth, studies have begun to address the question of how $\mathrm{T}$ cells get from the vasculature into the parenchyma and whether they remain within the parenchyma. A study in mice infected with Mtb and injected with an antibody specific to hematopoietic cells immediately before death showed that many of the antigen-specific $\mathrm{T}$ cells thought to have been in the lung parenchyma were actually in the vasculature ${ }^{63}$. These vascular T cells express CX3C chemokine receptor 1 CX3CR 1 and killer cell lectin-like receptor subfamily G member 1 (KLRG1) and have high expression of the transcription factor T-bet, suggesting that they are terminally differentiated effector cells ${ }^{63}$ (Fig. 2). When these $\mathrm{T}$ cells are transferred into infected recipient mice, they are unable to migrate into the parenchyma and are not efficient mediators of protection $^{63}$. In contrast, T cells within the parenchyma express CXCR3, migrate back to the lung and can mediate protection ${ }^{63}$. Importantly, antigen-specific memory $\mathrm{T}$ cells in people with latent infection (i.e., those who are controlling the infection) have a CXCR3 ${ }^{+} \mathrm{CCR}^{+}$ phenotype $^{7}$ and have multifunctional transcriptional profiles ${ }^{64}$. These observations have fundamentally changed the understanding of $\mathrm{T}$ cell activities in the lung. It is clearly not sufficient to induce large numbers of differentiated antigen-specific $T$ cells by vaccination if these $\mathrm{T}$ cells do not have the capacity to enter the lung parenchyma and then migrate to the correct location within the lung (Fig. 2). It is imperative that vaccine studies address the issue of $\mathrm{T}$ cell migratory capacity in addition to functional capacity to better define the $\mathrm{T}$ cell phenotype crucial in mediating protection.

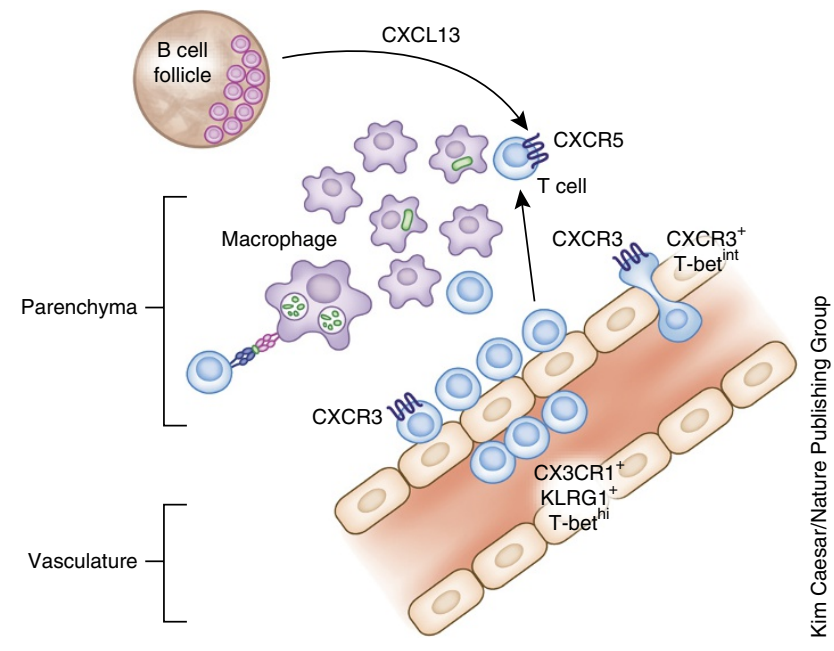

Interplay between inflammation and acquired immunity

Despite the presence of activated phagocytes and antigen-specific $\mathrm{T}$ cells, the initial lesion produced in response to infection can develop into a multilayered structure. The components of this structure vary depending on species and, indeed, within the lungs of infected individuals. In a detailed study of macaques it was apparent that individual granulomata could be initiated by a single Mtb bacterium but that the outcome of each lesion was different, even within the same host $^{65}$. The outcome was thought to depend on the rate of killing by the acquired response in each lesion and could result in either a progressive lesion or one that became sterile ${ }^{65}$. This internal variability again points to the fact that the generation of acquired immunity, while crucial, is not sufficient for control of disease. It is necessary that the acquired response be able to function efficiently within each individual granuloma.

What, then, affects the function of the acquired response in the granuloma (Fig. 3)? As discussed above, the presence of neutrophils is associated with the loss of containment, and other data have shown that regulatory $\mathrm{T}\left(\mathrm{T}_{\text {reg }}\right)$ cells can also limit the expression of immunity in the lung ${ }^{66,67}$. These $\mathrm{T}_{\text {reg }}$ cells seem to have a role in the delay of $\mathrm{T}$ cell priming after infection but are lost in an IL-12-dependent manner when mice are infected with the H37Rv laboratory strain of Mtb ${ }^{68}$ (Fig. 3a). In contrast, in mice infected with the W-Beijing clinical strain HN878, the number of $\mathrm{T}$ cells expressing the $\mathrm{T}_{\text {reg }}$-associated transcription factor Foxp 3 and IL-10 increases over time and coincides with the loss of T cells producing IFN- $\gamma^{69}$. Importantly, this temporal association of Foxp3-expressing $\mathrm{T}$ cells and the loss of bacterial control also occurs when mice vaccinated with BCG are infected with HN878 (ref. 70). These data suggest that $\mathrm{T}_{\text {reg }}$ cells have the capacity to compromise protective immunity even after vaccination, and this should be considered in vaccine development studies.

The cellular environment generated by Mtb is composed of inflammatory monocytes, macrophages, dendritic cells and neutrophils, in addition to $\mathrm{T}_{\text {reg }}$ cells. This myeloid-dominated environment can compromise the function of $\mathrm{T}$ cells in a variety of ways, and the impact may depend on the relative frequency of each type of cell (Fig. 3b). In this regard, Mtb seems to actively recruit permissive macrophages via a CCR2-CCL2 pathway ${ }^{28}$, and this recruitment may be influenced further by the amount of type I interferon expressed. Specifically, an artificial increase in the amount of type I interferon in chronically infected mice results in the recruitment of greater numbers of permissive macrophages, which are recruited in a CCR2-dependent manner ${ }^{71}$. Work has shown that type I interferon, IL-1 and prostaglandin $\mathrm{PGE}_{2}$ act 
Figure 3 The outcome of infection depends on the balance of several regulatory activities. (a) $\mathrm{T}_{\text {reg }}$ cells accumulate in the Mtb-infected animal and limit activation of effector T cells as well as the expression of acquired immunity in the lung. Induction and persistence of $T_{\text {reg }}$ cell populations differs for different strains of Mtb. Persistence of the $\mathrm{T}_{\text {reg }}$ cell population can be limited by IL-12. Nitric oxide also limits the accumulation of differentiated T cells in the mycobacterial granuloma. (b) Mtb specifically recruits permissive macrophages via CCL2 and CCR2, and this is augmented by the induction of type I interferon. Type I interferon also limits production of bioactive IL-1, whereas IL-1 limits type 1 interferon. IL-1 is required for optimal control of bacterial growth by driving $\mathrm{PGE}_{2}$ production, and $\mathrm{PGE}_{2}$ acts to drive control of bacterial growth by macrophages, probably by promoting apoptosis in these cells. In contrast, the anti-inflammatory lipid mediator $\mathrm{LXA}_{4}$ has a negative impact by driving necrosis of macrophages and thereby promoting bacterial growth. IL-1 can also drive damaging pathological consequences and is regulated by nitric oxide. The balance between these independent regulatory mechanisms may contribute to the stochastic nature of disease outcome after infection. +ve, positive effect; -ve, negative effect.

within a cross-regulatory network such that while type I interferon limits the production of IL-1 (refs. 72,73), IL-1 drives production of $\mathrm{PGE}_{2}$, which in turn regulates type I interferon ${ }^{74}$. The crucial action of IL- 1 in this model is to promote $\mathrm{PGE}_{2}$, which promotes the capacity of infected macrophages to limit bacterial growth ${ }^{74}$ (Fig. 3b). Published studies suggest that $\mathrm{PGE}_{2}$ acts to limit macrophage necrosis and promote apoptosis ${ }^{75,76}$, whereas the anti-inflammatory lipid mediator $\mathrm{LXA}_{4}$ promotes necrosis of infected macrophages, which increases bacterial growth ${ }^{75-77}$. The importance of the balance between pro- and anti-inflammatory eicosanoids has also been shown in studies where polymorphisms in leukotriene $\mathrm{A}_{4}$ hydrolase (which controls this balance) influence the outcome of disease and the efficacy of anti-inflammatory treatment ${ }^{78,79}$. This observation contributed substantially to the concept that in mycobacterial disease, both too little and too much inflammation can be bad for outcome, and it gave rise to the suggestion that intervention be focused on the inflammatory tendency of the individual being treated ${ }^{79}$.

Although Mtb bacteria are present throughout the granulomatous lesion, they do not occupy every macrophage in the lesion. Uninfected macrophages may present a barrier to the function of antigen-specific $\mathrm{T}$ cells in a variety of ways. The $\mathrm{T}$ cells could be limited in their migration toward infected macrophages by the physical barrier resulting from closely associated epithelioid macrophages surrounding the infected cells (Fig. 1). Similarly, secreted antigen may be presented by a variety of uninfected macrophages and act as a decoy for T cells. In addition, it is apparent that the activation of macrophages within the mycobacteria-induced lesion is detrimental to T cell accumulation. Mice that lack the capacity to generate the activated macrophage product nitric oxide, accumulate high numbers of T cells and have a lower bacterial burden $^{80}$. Not all activated $\mathrm{T}$ cells are equally susceptible to nitric oxide-the more differentiated $\mathrm{T}$ cells are more susceptible ${ }^{81}$ (Fig. 3a). Nitric oxide is also crucial in limiting the development of macrophage-mediated pathology. Importantly, it limits the activity of the NLRP3 inflammasome, which is required for IL-1-mediated innate inflammatory responses ${ }^{82}$ (Fig. 3b). The role of IL-1 as a mediator of damaging inflammation is in contrast to its function in driving $\mathrm{PGE}_{2}$ and controlling bacterial growth mentioned above ${ }^{74}$, but this serves to highlight the crucial importance of balance between protection and immunopathology in the outcome of Mtb infection (Fig. 3b).

\section{CONCLUSION}

The more investigators learn about the response to Mtb in the lung, the more complex it seems to become. Strong immune responses a

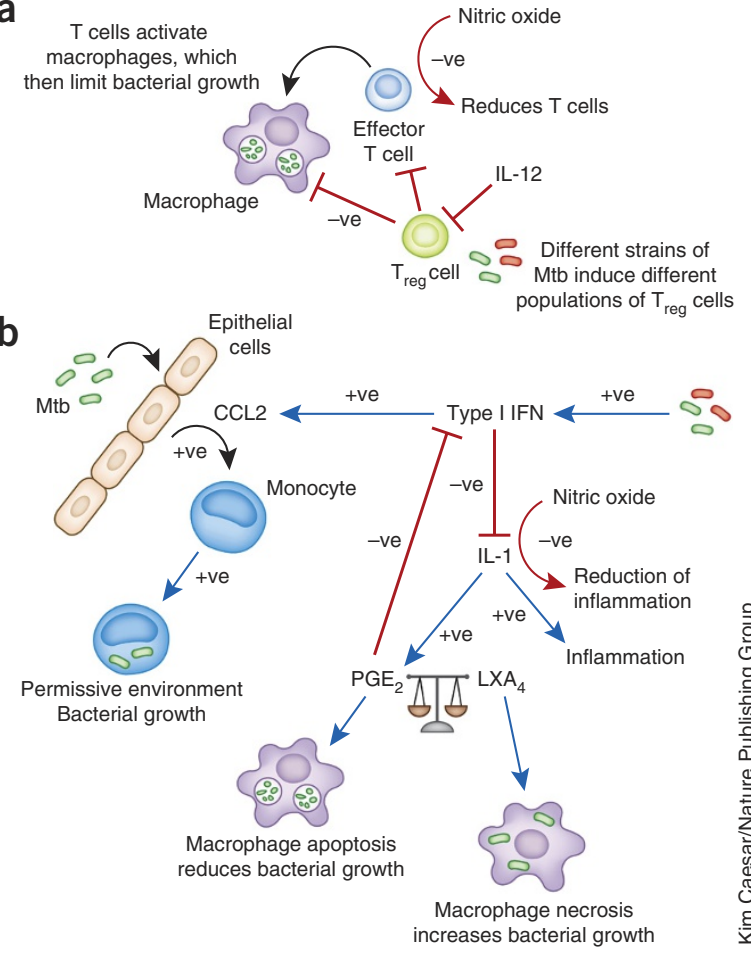

are induced and are required for survival; however, they are equally strongly regulated. If there is too much inflammation, the acquired response is compromised. If there is too little inflammation, bacteria grow without hindrance. The chances of infection leading to disease are low, and the crucial parameters mediating progression from infection to disease are unknown. The failure to identify those who are progressing to disease limits the capacity to intervene. Indeed, the enormous number of people currently infected makes it prohibitive to treat them all, so identifying those at risk in order to target intervention is essential. Defining the inflammatory tendency of the individual infected through prospective studies before disease develops should be highly informative.

\section{ACKNOWLEDGMENTS}

Supported by the Trudeau Institute, Inc., and the US National Institutes of Health NIAID (AI69121, AI73564 and AI67723 to A.M.C.; AI099661 R.T.R.).

\section{COMPETING FINANCIAL INTERESTS}

The authors declare no competing financial interests.

Reprints and permissions information is available online at http://www.nature.com/ reprints/index.html.

1. Trunz, B.B., Fine, P. \& Dye, C. Effect of BCG vaccination on childhood tuberculous meningitis and miliary tuberculosis worldwide: a meta-analysis and assessment of cost-effectiveness. Lancet 367, 1173-1180 (2006).

2. Colditz, G.A. et al. Efficacy of BCG vaccine in the prevention of tuberculosis. Metaanalysis of the published literature. J. Am. Med. Assoc. 271, 698-702 (1994).

3. Cooper, A.M. Cell mediated immune responses in tuberculosis. Annu. Rev. Immunol. 27, 393-422 (2009)

4. Tameris, M.D. et al. Safety and efficacy of MVA85A, a new tuberculosis vaccine, in infants previously vaccinated with BCG: a randomised, placebo-controlled phase 2b trial. Lancet 381, 1021-1028 (2013).

5. Gagneux, S. Host-pathogen coevolution in human tuberculosis. Phil. Trans. R. Soc Lond. B 367, 850-859 (2012).

6. Ford, C.B. et al. Use of whole genome sequencing to estimate the mutation rate of Mycobacterium tuberculosis during latent infection. Nat. Genet. 43, 482-486 (2011).

7. Lindestam Arlehamn, C.S. et al. Memory T cells in latent Mycobacterium tuberculosis infection are directed against three antigenic islands and largely contained in a CXCR+CCR+ Th1 Subset. PLoS Pathog. 9, e1003130 (2013). 
8. Comas, I. et al. Human T cell epitopes of Mycobacterium tuberculosis are evolutionarily hyperconserved. Nat. Genet. 42, 498-503 (2010).

9. Uplekar, S., Heym, B., Friocourt, V., Rougemont, J. \& Cole, S.T. Comparative genomics of Esx genes from clinical isolates of Mycobacterium tuberculosis provides evidence for gene conversion and epitope variation. Infect. Immun. 79, 4042-4049 (2011).

10. Ishikawa, E. et al. Direct recognition of the mycobacterial glycolipid, trehalose dimycolate, by C-type lectin Mincle. J. Exp. Med. 206, 2879-2888 (2009).

11. Werninghaus, K. et al. Adjuvanticity of a synthetic cord factor analogue for subunit Mycobacterium tuberculosis vaccination requires FcR $\gamma$-Syk-Card9-dependent innate immune activation. J. Exp. Med. 206, 89-97 (2009).

12. Schoenen, H. et al. Cutting edge: Mincle is essential for recognition and adjuvanticity of the mycobacterial cord factor and its synthetic analog trehalose-dibehenate. J. Immunol. 184, 2756-2760 (2010).

13. Miyake, Y. et al. C-type lectin MCL is an FcR $\gamma$-coupled receptor that mediates the adjuvanticity of mycobacterial cord factor. Immunity 38, 1050-1062 (2013).

14. Rao, V., Fujiwara, N., Porcelli, S. \& Glickman, M. Mycobacterium tuberculosis controls host innate immune activation through cyclopropane modification of a glycolipid effector molecule. J. Exp. Med. 201, 535-543 (2005).

15. Rao, V., Gao, F., Chen, B., Jacobs, W.J. \& Glickman, M. Trans-cyclopropanation of mycolic acids on trehalose dimycolate suppresses Mycobacterium tuberculosisinduced inflammation and virulence. J. Clin. Invest. 116, 1660-1667 (2006).

16. Dao, D.N. et al. Mycolic acid modification by the mmaA4 gene of $M$. tuberculosis modulates IL-12 production. PLoS Pathog. 4, e1000081 (2008).

17. Havlir, D.V. \& Barnes, P. Tuberculosis patients with human immunodeficiency virus infection. N. Engl. J. Med. 340, 367-373 (1999).

18. Rich, A.R. The Pathogenesis of Tuberculosis (Charles C Thomas, 1944).

19. Gold, M.C. et al. Human mucosal associated invariant $\mathrm{T}$ cells detect bacterially infected cells. PLoS Biol. 8, e1000407 (2010).

20. Rothchild, A.C., Jayaraman, P., Nunes-Alves, C. \& Behar, S.M. iNKT cell production of GM-CSF controls Mycobacterium tuberculosis. PLoS Pathog. 10, e1003805 (2014).

21. Chen, Z.W. Multifunctional immune responses of HMBPP-specific Vgamma2Vdelta2 T cells in M. tuberculosis and other infections. Cell. Mol. Immunol. 10, 58-64 (2013).

22. Azad, A.K., Sadee, W. \& Schlesinger, L.S. Innate immune gene polymorphisms in tuberculosis. Infect. Immun. 80, 3343-3359 (2012).

23. Filipe-Santos, O. et al. Inborn errors of IL-12/23- and IFN- $\gamma$-mediated immunity: molecular, cellular, and clinical features. Semin. Immunol. 18, 347-361 (2006).

24. Filipe-Santos, 0 . et al. X-linked susceptibility to mycobacteria is caused by mutations in NEMO impairing CD40-dependent IL-12 production. J. Exp. Med. 203, 1745-1759 (2006).

25. Arcos, J. et al. Human lung hydrolases delineate Mycobacterium tuberculosismacrophage interactions and the capacity to control infection. J. Immunol. 187 372-381 (2011).

26. Leemans, J.C. et al. Depletion of alveolar macrophages exerts protective effects in pulmonary tuberculosis in mice. J. Immunol. 166, 4604-4611 (2001).

27. Leemans, J.C. et al. Macrophages play a dual role during pulmonary tuberculosis in mice. J. Infect. Dis. 191, 65-74 (2005).

28. Cambier, C.J. et al. Mycobacteria manipulate macrophage recruitment through coordinated use of membrane lipids. Nature 505, 218-222 (2014).

29. Martineau, A.R. et al. Neutrophil-mediated innate immune resistance to mycobacteria. J. Clin. Invest. 117, 1988-1994 (2007).

30. Casanova, J.L. \& Abel, L. The genetic theory of infectious diseases: a brief history and selected illustrations. Annu. Rev. Genomics Hum. Genet. 14, 215-243 (2013).

31. Vanden Driessche, K., Persson, A., Marais, B.J., Fink, P.J. \& Urdahl, K.B. Immune vulnerability of infants to tuberculosis. Clin. Dev. Immunol. 2013, 781320 (2013).

32. Zhang, M. et al. T cell cytokine responses in persons with tuberculosis and human immunodeficiency virus infection. J. Clin. Invest. 94, 2435-2442 (1994).

33. Chackerian, A.A., Alt, J., Perera, T., Dascher, C. \& Behar, S. Dissemination of Mycobacterium tuberculosis is influenced by host factors and precedes the initiation of T-cell immunity. Infect. Immun. 70, 4501-4509 (2002).

34. Reiley, W.W. et al. ESAT-6-specific CD4 T cell responses to aerosol Mycobacterium tuberculosis infection are initiated in mediastinal lymph nodes. Proc. Natl. Acad. Sci. USA 105, 10961-10966 (2008).

35. Wolf, A.J. et al. Initiation of the adaptive immune response to Mycobacterium tuberculosis depends on antigen production in the local lymph node, not the lungs. J. Exp. Med. 205, 105-115 (2008).

36. Orme, I.M. A new unifying theory of the pathogenesis of tuberculosis. Tuberculosis (Edinb.) 94, 8-14 (2014).

37. Ramakrishnan, L. Revisiting the role of the granuloma in tuberculosis. Nat. Rev. Immunol. 12, 352-366 (2012)

38. Schreiber, H.A. et al. Inflammatory dendritic cells migrate in and out of transplanted chronic mycobacterial granulomas in mice. J. Clin. Invest. 121, 3902-3913 (2011)

39. Davis, J.M. \& Ramakrishnan, L. The role of the granuloma in expansion and dissemination of early tuberculous infection. Cell 136, 37-49 (2009).

40. Egen, J.G. et al. Macrophage and T cell dynamics during the development and disintegration of mycobacterial granulomas. Immunity 28, 271-284 (2008).

41. Egen, J.G. et al. Intravital imaging reveals limited antigen presentation and T cell effector function in mycobacterial granulomas. Immunity 34, 807-819 (2011).
42. Bhatt, K., Hickman, S.P. \& Salgame, P. Cutting edge: a new approach to modeling early lung immunity in murine tuberculosis. J. Immunol. 172, 2748-2751 (2004).

43. Khader, S.A. et al. Interleukin $12 \mathrm{p} 40$ is required for dendritic cell migration and $\mathrm{T}$ cell priming after Mycobacterium tuberculosis infection. J. Exp. Med. 203 1805-1815 (2006).

44. Robinson, R.T. et al. Mycobacterium tuberculosis infection induces il12rb1 splicing to generate a novel IL-12R $\beta 1$ isoform that enhances DC migration. J. Exp. Med. 207, 591-605 (2010)

45. Keeton, $\mathrm{R}$. et al. Soluble TNFRp75 regulates host protective immunity against Mycobacterium tuberculosis. J. Clin. Invest. 124, 1537-1551 (2014).

46. Samstein, M. et al. Essential yet limited role for CCR2 ${ }^{+}$inflammatory monocytes during Mycobacterium tuberculosis-specific T cell priming. eLife 2, e01086 (2013).

47. Gallegos, A.M., Pamer, E. \& Glickman, M. Delayed protection by ESAT-6-specific effector $\mathrm{CD}^{+} \mathrm{T}$ cells after airborne M. tuberculosis infection. J. Exp. Med. 205, 2359-2368 (2008)

48. Khader, S.A. et al. IL-23 and IL-17 in establishment of protective pulmonary CD4 $T$ cell responses upon vaccination and during Mycobacterium tuberculosis challenge. Nat. Immunol. 8, 369-377 (2007).

49. Gonzalez-Juarrero, M. \& Orme, I. Characterization of murine lung dendritic cells infected with Mycobacterium tuberculosis. Infect. Immun. 69, 1127-1133 (2001).

50. Wolf, A.J. et al. Mycobacterium tuberculosis infects dendritic cells with high frequency and impairs their function in vivo. J. Immunol. 179, 2509-2519 (2007).

51. Sköld, M. \& Behar, S.M. Tuberculosis triggers a tissue dependent program of differentiation and acquisition of effector functions by circulating monocytes. J. Immunol. 181, 6349-6360 (2008).

52. Cooper, A.M., Adams, L.B., Dalton, D.K., Appelberg, R. \& Ehlers, S. IFN- $\gamma$ and NO in mycobacterial disease: new jobs for old hands. Trends Microbiol. 10, 221-226 (2002).

53. Nandi, B. \& Behar, S.M. Regulation of neutrophils by interferon- $\gamma$ limits lung inflammation during tuberculosis infection. J. Exp. Med. 208, 2251-2262 (2011).

54. Desvignes, L. \& Ernst, J.D. Interferon- $\gamma$-responsive nonhematopoietic cells regulate the immune response to Mycobacterium tuberculosis. Immunity 31, 974-985 (2009).

55. Eum, S.Y. et al. Neutrophils are the predominant infected phagocytic cells in the airways of patients with active pulmonary TB. Chest 137, 122-128 (2010).

56. Berry, M.P. et al. An interferon-inducible neutrophil-driven blood transcriptiona signature in human tuberculosis. Nature 466, 973-977 (2010)

57. Srivastava, S. \& Ernst, J.D. Cutting edge: direct recognition of infected cells by CD4 T cells is required for control of intracellular Mycobacterium tuberculosis in vivo. J. Immunol. 191, 1016-1020 (2013).

58. Khader, S.A. et al. In a murine tuberculosis model, the absence of homeostatic chemokines delays granuloma formation and protective immunity. J. Immunol. 183, 8004-8014 (2009)

59. Ulrichs, T. et al. Human tuberculous granulomas induce peripheral lymphoid folliclelike structures to orchestrate local host defence in the lung. J. Pathol. 204, 217-228 (2004).

60. Kahnert, A. et al. Mycobacterium tuberculosis triggers formation of lymphoid structure in murine lungs. J. Infect. Dis. 195, 46-54 (2007).

61. Slight, S.R. et al. CXCR5 $5^{+} \mathrm{T}$ helper cells mediate protective immunity agains tuberculosis. J. Clin. Invest. 123, 712-726 (2013).

62. Khader, S.A. et al. IL-23 is required for long-term control of Mycobacterium tuberculosis and B cell follicle formation in the infected lung. J. Immunol. 187, 5402-5407 (2011).

63. Sakai, S. et al. Cutting edge: control of Mycobacterium tuberculosis infection by a subset of lung parenchyma-homing CD4 T cells. J. Immunol. 192, 2965-2969 (2014).

64. Arlehamn, C.L. et al. Transcriptional profile of tuberculosis antigen-specific T cells reveals novel multifunctional features. J. Immunol. 193, 2931-2940 (2014).

65. Lin, P.L. et al. Sterilization of granulomas is common in active and latent tuberculosis despite within-host variability in bacterial killing. Nat. Med. 20, 75-79 (2014).

66. Scott-Browne, J. et al. Expansion and function of Foxp3-expressing T regulatory cells during tuberculosis. J. Exp. Med. 204, 2159-2169 (2007).

67. Shafiani, S., Tucker-Heard, G., Kariyone, A., Takatsu, K. \& Urdahl, K.B. Pathogenspecific regulatory $T$ cells delay the arrival of effector $T$ cells in the lung during early tuberculosis. J. Exp. Med. 207, 1409-1420 (2010)

68. Shafiani, S. et al. Pathogen-specific Treg cells expand early during Mycobacterium tuberculosis infection but are later eliminated in response to interleukin-12. Immunity 38, 1261-1270 (2013).

69. Ordway, D. et al. The hypervirulent Mycobacterium tuberculosis strain HN878 induces a potent $\mathrm{TH} 1$ response followed by rapid down-regulation. J. Immunol. 179, 522-531 (2007).

70. Ordway, D.J. et al. Mycobacterium bovis BCG-mediated protection against W-Beijing strains of Mycobacterium tuberculosis is diminished concomitant with the emergence of regulatory T cells. Clin. Vaccine Immunol. 18, 1527-1535 (2011).

71. Antonelli, L.R. et al. Intranasal poly-IC treatment exacerbates tuberculosis in mice through the pulmonary recruitment of a pathogen-permissive monocyte/macrophage population. J. Clin. Invest. 120, 1674-1682 (2010). 
72. Mayer-Barber, K.D. et al. Innate and adaptive interferons suppress IL- $1 \alpha$ and IL-1 $\beta$ production by distinct pulmonary myeloid subsets during Mycobacterium tuberculosis infection. Immunity 35, 1023-1034 (2011).

73. Novikov, A. et al. Mycobacterium tuberculosis triggers host type I IFN signaling to regulate IL-1 $\beta$ production in human macrophages. J. Immunol. 187, 2540-2547 (2011).

74. Mayer-Barber, K.D. et al. Host-directed therapy of tuberculosis based on interleukin1 and type I interferon crosstalk. Nature 511, 99-103 (2014).

75. Chen, M. et al. Lipid mediators in innate immunity against tuberculosis: opposing roles of PGE2 and LXA4 in the induction of macrophage death. J. Exp. Med. 205, 2791-2801 (2008).

76. Divangahi, M., Desjardins, D., Nunes-Alves, C., Remold, H.G. \& Behar, S.M. Eicosanoid pathways regulate adaptive immunity to Mycobacterium tuberculosis. Nat. Immunol. 11, 751-758 (2010).
77. Divangahi, M. et al. Mycobacterium tuberculosis evades macrophage defenses by inhibiting plasma membrane repair. Nat. Immunol. 10, 899-906 (2009).

78. Tobin, D.M. et al. The Ita4h locus modulates susceptibility to mycobacterial infection in zebrafish and humans. Cell 140, 717-730 (2010).

79. Tobin, D.M. et al. Host genotype-specific therapies can optimize the inflammatory response to mycobacterial infections. Cell 148, 434-446 (2012).

80. Gomes, M.S., Florido, M., Pais, T.F. \& Appelberg, R. Improved clearance of Mycobacterium avium upon disruption of the inducible nitric oxide synthase gene. J. Immunol. 162, 6734-6739 (1999).

81. Pearl, J.E. et al. Nitric oxide inhibits the accumulation of CD4+CD44hiTbet ${ }^{+} \mathrm{CD} 69$ lo T cells in mycobacterial infection. Eur. J. Immunol. 42, 3267-3279 (2012).

82. Mishra, B.B. et al. Nitric oxide controls the immunopathology of tuberculosis by inhibiting NLRP3 inflammasome-dependent processing of IL-1ß. Nat. Immunol. 14, 52-60 (2013). 